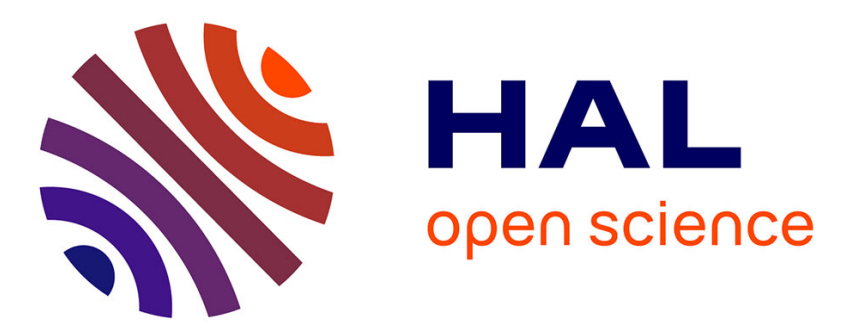

\title{
Propriétés électroniques des agrégats CrpCn et FepCn mises en évidence par spectrométrie de masse
}

\author{
P. Joyes, M. Leleyter, A. Hoareau
}

\section{To cite this version:}

P. Joyes, M. Leleyter, A. Hoareau. Propriétés électroniques des agrégats CrpCn et FepCn mises en évidence par spectrométrie de masse. Journal de Physique Lettres, 1979, 40 (6), pp.131-135. 10.1051/jphyslet:01979004006013100 . jpa-00231590

\section{HAL Id: jpa-00231590 https://hal.science/jpa-00231590}

Submitted on 1 Jan 1979

HAL is a multi-disciplinary open access archive for the deposit and dissemination of scientific research documents, whether they are published or not. The documents may come from teaching and research institutions in France or abroad, or from public or private research centers.
L'archive ouverte pluridisciplinaire HAL, est destinée au dépôt et à la diffusion de documents scientifiques de niveau recherche, publiés ou non, émanant des établissements d'enseignement et de recherche français ou étrangers, des laboratoires publics ou privés. 


\title{
Propriétés électroniques des agrégats $\mathrm{Cr}_{p} \mathrm{C}_{n}$ et $\mathrm{Fe}_{p} \mathrm{C}_{n}$ mises en évidence par spectrométrie de masse
}

\author{
P. Joyes, M. Leleyter $\left(^{*}\right)$ \\ Laboratoire de Physique des Solides (**), Bâtiment 510, Université Paris-Sud, F-91405 Orsay, France
}

et A. Hoareau

Département de Physique des Matériaux (**), Université Lyon I, 43, bd du 11-Novembre-1918, F-69621 Villeurbanne, France

(Reçu le 27 novembre 1978, accepté le 23 janvier 1979)

\begin{abstract}
Résumé. - Les intensités d'émission (par une source à étincelles ou par émission ionique secondaire) des ions $\mathrm{Fe}_{p} \mathrm{C}_{n}^{+}, \mathrm{Cr}_{p} \mathrm{C}_{n}^{+}(p=1$ à 4$)$ et $\mathrm{CrC}_{n}^{-}$, mesurées au spectrographe de masse, présentent un comportement en dents de scie si $p=1$ et $3(\mathrm{Fe})$; si $p=1$ et $4(\mathrm{Cr})$ suivant la parité du nombre $n$ d'atomes de carbone.

Ce phénomène peut être interprété dans le cas $p=1$ à partir du modèle de Pitzer et Clementi en supposant que l'élément de transition fournit un nombre variable suivant l'élément d'électrons $\pi$ à la bande $\pi$ de l'amas, hypothèses qui devront être vérifiées par un calcul de chimie quantique. Ce modèle permet aussi d'interpréter les résultats relatifs à $\mathrm{ZrC}_{n}^{+}$.

Abstract. - The emission intensities (in spark source or secondary ionic emission experiments) of $\mathrm{Fe}_{p} \mathrm{C}_{n}^{+}$, $\mathrm{Cr}_{p} \mathrm{C}_{n}^{+}(p=1$ to 4$)$ and $\mathrm{CrC}_{n}^{-}$clusters, measured by mass spectrometry show an alternating behaviour if $p=1$ and $3(\mathrm{Fe})$ or $p=1$ and $4(\mathrm{Cr})$ according to the parity of the number $n$ of carbon atoms.

This phenomenon can be interpreted in the simpler case $p=1$ with the Pitzer and Clementi model by supposing that the transition element gives electrons to the cluster $\pi$ band (the number of these electrons depends on the element). These hypotheses should be checked by a quantum chemical calculation. Moreover, this model allows interpretation of the results for $\mathrm{ZrC}_{n}^{+}$ions.
\end{abstract}

La physique des petits amas prend à l'heure actuelle une importance croissante. Les techniques de production d'amas sont multiples; nous parlerons ici des résultats de deux d'entre elles : l'émission ionique secondaire (E.I.S.) où les agrégats sont émis à partir de cibles solides soumises à l'impact d'ions primaires et les sources à étincelles.

Dans ces deux techniques, la proportion de particules moléculaires émises directement sous forme d'ions est importante et l'analyse par spectrométrie de masse des intensités des diverses espèces ionisées donne des informations intéressantes.

Ainsi, lorsque l'on étudie un élément du groupe I-A (Li par exemple) par E.I.S. ou par source à étincelles, les courants en ions $\mathrm{Li}_{n}^{+}$correspondant aux amas à nombre impair d'atomes $I\left(\mathrm{Li}_{2 k \pm 1}^{+}\right)$sont systématiquement plus élevés que ceux qui correspondent à un nombre pair $I\left(\mathrm{Li}_{2 k}^{+}\right)[1,2]$.

$\left({ }^{*}\right)$ Aussi : Département de Physique, Faculté des Sciences, Université de Picardie, 33, rue Saint-Leu, F-80000 Amiens, France. (**) Laboratoires associés au C.N.R.S. (LA 2 et 172).
Les études faites sur cette question $[1,3]$ ont permis de conclure que, dans ce type d'expérience, les intensités d'émission dépendaient surtout des caractéristiques électroniques des amas émis. Le comportement fut résumé par la règle qualitative suivante :

«A une intensité relative plus élevée de l'ion $\mathrm{X}_{n}^{+}$ (resp. $\mathrm{X}_{n}^{-}$) correspond une énergie d'ionisation (resp. une affinité électronique) plus élevée de la molécule $\mathrm{X}_{n}$.»

Cette règle intuitivement raisonnable a été vérifiée sur de nombreux exemples [4-8]. Vu son caractère approché, il ne faut cependant pas espérer s'en servir lorsque les différences entre intensités sont faibles. Il est nécessaire, au contraire, que l'effet expérimental soit net comme par exemple le comportement en dents de scie des ions $\mathrm{Li}_{n}^{+}$. Dans les expériences que nous voulons analyser ici, c'est justement à de tels comportements que nous avons affaire et nous pourrons donc avoir recours à la règle précédente.

On peut d'ailleurs espérer que cette correspondance (intensité $\Leftrightarrow$ caractéristique électronique) devienne 
plus quantitative dans un proche avenir, du moins pour l'E.I.S. Il semble en effet possible d'introduire les paramètres électroniques des agrégats dans les théories de l'émission de paquets d'atomes, théories qui connaissent actuellement un grand développement $[9,10]$.

1. Résultats expérimentaux. - Nous analysons dans cet article deux séries de résultats. Nous rappelons d'abord ceux obtenus avec une source à étincelles sur les amas $\mathrm{Fe}_{p} \mathrm{C}_{n}^{+}$par Vidal et al. [11] (Fig. 1)

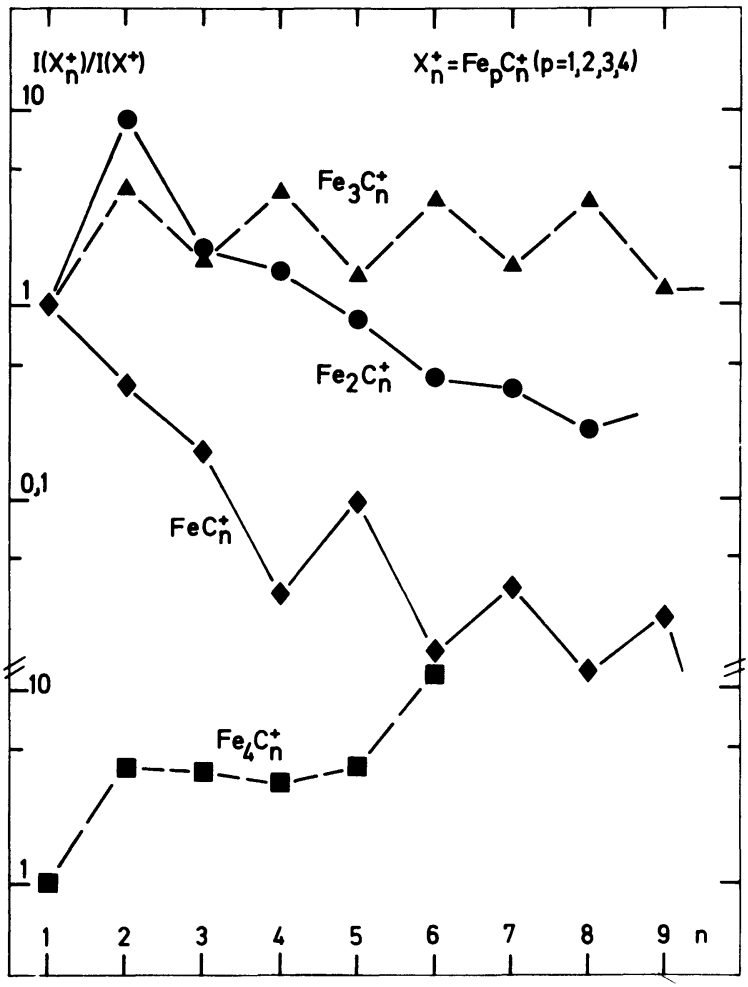

Fig. 1. - Intensités relatives des ions $\mathrm{Fe}_{p} \mathrm{C}_{n}^{+}$obtenus par spectrométrie de masse de source à étincelles en fonction du nombre $n$ d'atomes de carbone (mesures de G. Vidal et al. [11]).

[Relative intensities of $\mathrm{Fe}_{p} \mathrm{C}_{n}^{+}$ions obtained by spark source mass spectrometry versus the number $n$ of carbon atoms (from G. Vidal et al. [11]).]

et nous présentons par ailleurs nos propres résultats obtenus en E.I.S. à partir du composé $\mathrm{Cr}_{7} \mathrm{C}_{3}$ pour les ions $\mathrm{Cr}_{p} \mathrm{C}_{n}^{+}$et $\mathrm{CrC}_{n}^{-}$(Figs. $2 a$ et $2 b$ ).

De l'examen de la figure 1 , on retiendra surtout que pour $p=1$ ou 3 , il apparaît des alternances extrêmement nettes selon la parité de $n$ dans les intensités $I\left(\mathrm{Fe}_{p} \mathrm{C}_{n}^{+}\right)$. Les figures $2 a$ et $2 b$ montrent de même des dents de scie dans les intensités $I\left(\mathrm{Cr}_{p} \mathrm{C}_{n}^{+}\right)$ pour $p=1$ ou 4 , et dans celles de $\mathrm{CrC}_{n}^{-}$. On pourra d'ailleurs remarquer que les alternances des ions $\mathrm{FeC}_{n}^{+}$sont renversées par rapport à celles des ions $\mathrm{CrC}_{n}^{+}$

2. Discussion et interprétation proposée. - Les molécules $\mathrm{C}_{n}$ ont déjà été étudiées. La structure de niveaux proposée par Pitzer et Clementi [12] qui

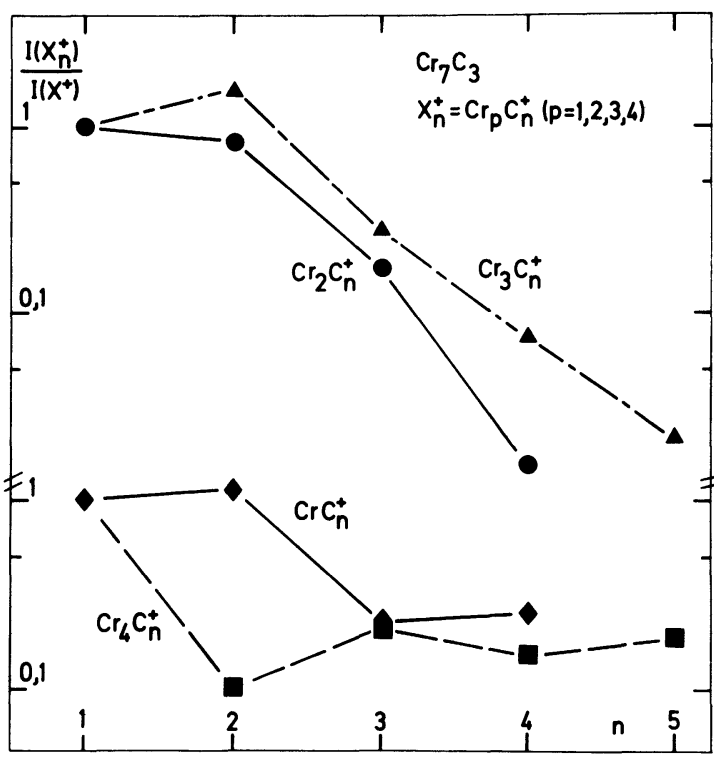

a)

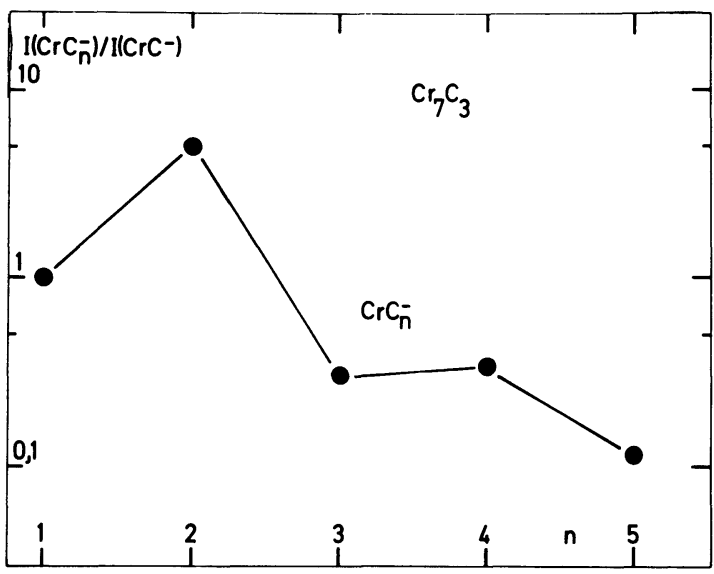

b)

Fig. 2. - E.I.S. de $\mathrm{Cr}_{7} \mathrm{C}_{3}$ (ions primaires $\mathrm{Ar}^{+}$de $6,5 \mathrm{keV}$ ). a) Intensités relatives des ions $\mathrm{Cr}_{p} \mathrm{C}_{n}^{+}$en fonction du nombre $n$ d'atomes de carbone. $b$ ) Intensités relatives des ions $\mathrm{CrC}_{n}^{-}$en fonction de $n$.

[S.I.M.S. of $\mathrm{Cr}_{7} \mathrm{C}_{3}$ (primary ions : $6.5 \mathrm{keV} \mathrm{Ar}{ }^{+}$). a) Relative intensities of $\mathrm{Cr}_{p} \mathrm{C}_{n}^{+}$ions versus the number $n$ of carbon atoms. b) Relative intensities of $\mathrm{CrC}_{n}^{-}$ions versus $n$.]

rend compte à la fois de la prédominance des agrégats à nombre impair d'atomes dans la vapeur [13] et des alternances des affinités électroniques des $C_{n}[14]$ (affinités électroniques plus élevées pour $n$ pair) est la suivante : par ordre croissant d'énergie, on trouve :

- une bande $\operatorname{sp} \sigma$ liante contenant $2 n-2$ électrons,

- 2 niveaux $\sigma$ de bout de chaîne avec 4 électrons,

- une bande $\pi$ comportant $2 n-2$ électrons dont le dernier niveau occupé est complet lorsque $n$ est impair, et incomplet lorsque $n$ est pair,

- enfin une bande $\operatorname{sp} \sigma$ antiliante vide.

Il n'existe pas d'études théoriques sur les agglomérats $\mathrm{Fe}_{p} \mathrm{C}_{n}$ ou $\mathrm{Cr}_{p} \mathrm{C}_{n}$. La liaison $\mathrm{Ni}-\mathrm{CO}$ a cependant été analysée dans le cadre d'un travail plus général sur les complexes de nickel-carbonyle [15]. 
Les résultats montrent que le nickel a tendance à former deux orbitales hybrides $\sigma$ à partir des orbitales atomiques $3 \mathrm{~d} \sigma$ et $4 \mathrm{~s} \sigma$; l'une, très fortement centrée sur l'atome, conserve deux électrons, l'autre se lie à l'orbitale de bout de chaîne du carbone, ce qui se traduit par l'apparition d'un état liant peuplé par les deux électrons qui occupaient l'orbitale de bout de chaîne et un état antiliant vide. Par ailleurs, le niveau $\mathrm{d} \delta$ du nickel conserve 4 électrons.

Ainsi, sur les 10 électrons externes du nickel, il reste 4 électrons $\pi(10-6)$ à répartir sur la bande $\pi$ constituée par la combinaison des niveaux $\pi$ de $\mathrm{CO}$ et $\mathrm{d} \pi$ de Ni.

Si l'on suppose que le fer, dont les niveaux d'énergie atomiques sont très proches de ceux du nickel, $a$ un comportement identique à celui du nickel, et que la molécule $C_{n}$, qui possède, elle aussi, une structure linéaire et des niveaux de bout de chaîne, a un comportement voisin de celui de $\mathrm{CO}$, on peut avancer certaines hypothèses sur la structure des agrégats $\mathrm{FeC}_{n}$. Dans ce schéma, on peut supposer que le fer se lie par une liaison $\sigma$ à la chaîne, que par ailleurs, il conserve sur des niveaux $\sigma$ et $\delta$ à fort caractère atomique 6 électrons, donc qu'il reste 2 électrons $\pi$ à répartir dans la bande $\pi$. Ces électrons s'ajoutent

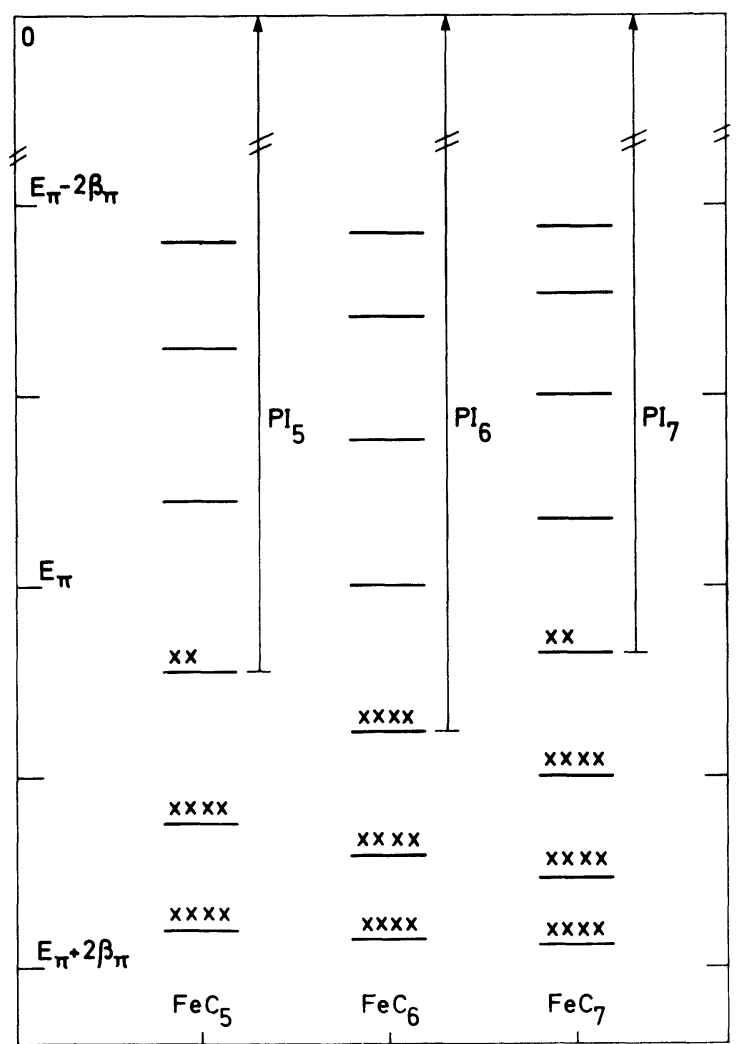

Fig. 3. - Niveaux d'énergie de la bande $\pi$ (dans l'approximation de Hückel [20]) des agrégats $\mathrm{FeC}_{n}$ (Fig. $3 a$ ) et $\mathrm{CrC}_{n}$ (Fig. 3b) et populations électroniques de ces niveaux (énergies en unités $\beta_{\pi}$ : on a pris un seul $\beta_{\pi}$ pour décrire la liaison $\mathrm{Fe}-\mathrm{C}$ et la liaison $\mathrm{C}-\mathrm{C}$ ). $E_{\pi}$ représente le milieu de la bande. On a matérialisé l'énergie d'ionisation $P I_{n}$ (flèches en traits pleins) et pour les amas $\mathrm{CrC}_{n}$ l'affinité électronique $A E_{n}$ (flèches en tirets). aux $2 n-2$ électrons $\pi$ de $C_{n}$. Pour $n$ pair, le dernier niveau $\pi$, qui était incomplet pour $\mathrm{C}_{n}$, devient complet pour $\mathrm{FeC}_{n}$, l'effet inverse se produisant pour $n$ impair (Fig. 3a). Dans ce dernier cas, on peut donc s'attendre à avoir une énergie d'ionisation $P I_{n}$ systématiquement plus faible que pour $n$ pair (Fig. $3 a$ ). Si l'on se réfère alors à la règle de correspondance énoncée plus haut, on voit que l'on comprend le résultat expérimental, c'est-à-dire pourquoi les intensités d'émission des ions $\mathrm{FeC}_{n}^{+}$sont systématiquement plus élevées pour $n$ impair.

Le schéma précédent peut s'étendre aux molécules $\mathrm{CrC}_{n}$. Il conduit à prévoir un renversement du comportement des énergies d'ionisation $P I_{n}$ puisque maintenant le nombre des électrons $\pi$ apportés par le chrome est nul (6-6) (Fig. 3b). A ce renversement doit correspondre celui des intensités d'émission mesurées, phénomène qui est effectivement observé.

Par ailleurs, l'examen de la même figure $3 b$ montre que les affinités électroniques $A E_{n}$ sont plus élevées pour $n$ pair que pour $n$ impair, ce qui correspond bien aux résultats d'expérience.

Il convient de faire deux remarques sur la discussion précédente. Si l'on s'en tenait au schéma décrit plus haut, on s'attendrait à observer des courants plus

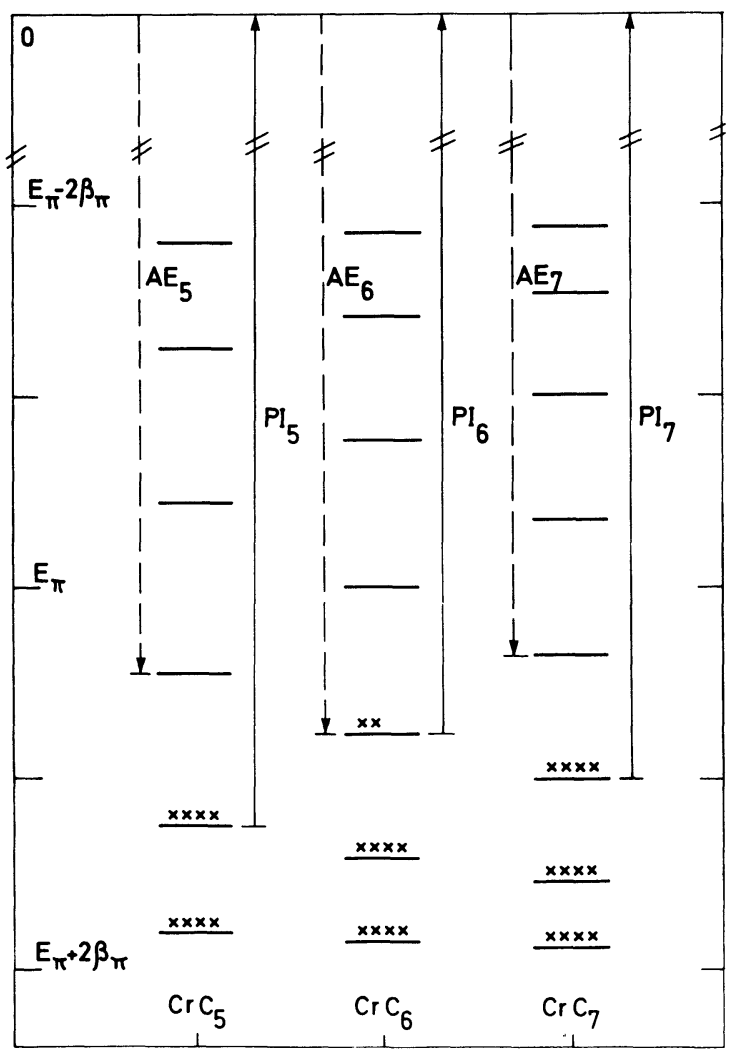

[Energy levels in the $\pi$ band (Hückel approximation [20]) of the $\mathrm{FeC}_{n}$ (Fig. 3a) and $\mathrm{CrC}_{n}$ (Fig. 3b) aggregates and electronic population of these levels (energies are in $\beta_{\pi}$ units; only one $\beta_{\pi}$ has been taken to describe the two $\mathrm{Fe}-\mathrm{C}$ and $\mathrm{C}-\mathrm{C}$ bondings). $\mathrm{E}_{\pi}$ is the middle of the $\pi$ band. Ionization energies $P I_{n \circ}$ and for $\mathrm{CrC}_{n}$ clusters, electron affinities $A E_{n}$ are shown (respectively arrows in solid lines or in dashed lines).] 
intenses pour les ions $\mathrm{C}_{n}^{+}$et $\mathrm{C}_{n}^{-}$à nombre pair d'atomes; c'est bien le cas pour $\mathrm{C}_{n}^{-}[7,8,14]$, mais pas pour $\mathrm{C}_{n}^{+}$. La raison de ceci provient du fait que si notre modèle nous permet de dire que les énergies d'ionisation sont plus faibles pour telle parité que pour telle autre, il ne nous permet pas de chiffrer l'importance de l'alternance pair-impair. Seul un calcul plus élaboré pourra nous mettre à même de fixer ce point. Il est à prévoir que pour les molécules $C_{n}$, les alternances de l'énergie d'ionisation disparaissent presque complètement, ce que semble bien montrer à la fois la mesure directe de ces énergies [16] (C : $11,3 \mathrm{eV}, \mathrm{C}_{2}: 13 \mathrm{eV}, \mathrm{C}_{3}$ : de 12,6 à $14,4 \mathrm{eV}$, $\mathrm{C}_{4}: 12,6 \mathrm{eV}, \mathrm{C}_{5}: 12,7 \mathrm{eV}, \mathrm{C}_{6}: 12,5 \mathrm{eV}$ ), et les mesures d'abondance relative des ions en E.I.S. ([7] et réf. in [7]).

On peut aussi remarquer que nous n'avons pas tenu compte des propriétés magnétiques des éléments métalliques considérés. Pour appuyer ce choix, notons d'abord que cette même hypothèse a déjà été utilisée par Anderson [17] qui, pour $\mathrm{Fe}_{2}$ et $\mathrm{Cr}_{2}$, propose des configurations moléculaires à couche complète (respectivement $\sigma_{\mathrm{g}}^{2} \pi_{\mathrm{u}}^{4} \delta_{\mathrm{g}}^{4} \sigma_{\mathrm{g}}^{\prime 2} \delta_{\mathrm{u}}^{4}, \quad \sigma_{\mathrm{g}}^{2} \pi_{\mathrm{u}}^{4}{\sigma_{\mathrm{g}}^{\prime 2}}^{4}{ }_{\mathrm{g}}^{4}$ ). Par ailleurs, on peut dire que dans certaines combinaisons chimiques, le fer perd son caractère magnétique ; c'est le cas de certains complexes comme par exemple le ferrocène $\mathrm{Fe}\left(\mathrm{C}_{5} \mathrm{H}_{2}\right)_{2}$ qui est diamagnétique. Ceci laisse à penser que même s'il existe pour les molécules étudiées des états magnétiques d'énergie comparable, l'état non magnétique analysé est une représentation possible de la molécule.

Le résultat expérimental pour les amas $\mathrm{Fe}_{3} \mathrm{C}_{n}^{+}$ montre un renversement d'alternances par rapport à $\mathrm{FeC}_{n}^{+}$. Pour tenter d'interpréter ceci, supposons la molécule linéaire et les 3 atomes de fer ajoutés du même côté. Si l'on attribue les alternances aux électrons $\pi$ comme plus haut, il est nécessaire que le nombre d'électrons $\pi$ apportés par les trois atomes de fer à la bande $\pi$ soit un multiple de 4 ; la situation la plus vraisemblable est que ce nombre soit 4 . En admettant alors que comme précédemment, il y a 4 électrons d par atome de fer, cela revient à attribuer 8 électrons $\sigma$ sur les 24 électrons externes du fer (24-4 $\pi-12 \delta-8)$. On peut alors a posteriori s'assurer que cette situation est assez logique; il y a en effet en moyenne 3 électrons $\sigma$ par atome de fer $\left(\frac{8+1}{3}\right.$, car il faut ajouter l'électron $\sigma$ que le fer partage avec la chaîne de carbone qui est donneur), donc le même nombre d'électrons $\sigma$ par atome de fer que dans les amas $\mathrm{FeC}_{n}^{+}$.

Il n'en reste pas moins que le schéma précédent comporte un grand nombre d'hypothèses. On pourrait de même essayer de construire un modèle permettant de rendre compte du renversement des alternances dans le passage de $\mathrm{CrC}_{n}^{+}$à $\mathrm{Cr}_{4} \mathrm{C}_{n}^{+}$. Dans la mesure où pour cette dernière molécule, il y a 4 atomes métalliques et que la situation se complique lorsque le nombre d'atomes de transition croît, ce modèle aurait un caractère encore plus hypothétique que celui proposé pour $\mathrm{Fe}_{3} \mathrm{C}_{n}^{+}$. Pour cette raison, nous attendrons les premières indications des calculs de chimie quantique sur la liaison $\mathrm{C}-\mathrm{Cr}$ pour avancer une explication dont nous pensons toutefois qu'elle relève aussi du caractère complet ou non de la bande $\pi$. Nous ajouterons encore une remarque sur le spin des molécules analysées. L'étude des complexes de transition montre qu'un même élément métallique peut être dans un état de bas-spin ou de spin-élevé selon la nature du ligand [18]. Il se peut donc que pour les amas du type $\mathrm{Cr}_{4} \mathrm{C}_{n}^{+}$(et même $\mathrm{Fe}_{3} \mathrm{C}_{n}^{+}$), l'hypothèse de non-magnétisme, qui semble valable pour $\mathrm{FeC}_{n}^{+}$ et $\mathrm{CrC}_{n}^{+}$, doive être remise en cause.

3. Conclusion. - Ces résultats ouvrent la voie à plusieurs autres études théoriques ou expérimentales.

Si l'on prend ce dernier point de vue, on peut étendre les conclusions de notre schéma et prévoir que l'on devrait observer un autre renversement d'alternances lorsque l'on passe du fer au nickel qui a 2 électrons de valence de plus $\left(\mathrm{NiC}_{n}^{+}\right.$plus intenses pour $n$ pair). D'autres expériences sur les ions négatifs devraient, comme nous l'avons vérifié pour $\mathrm{CrC}_{n}^{-}$ par rapport à $\mathrm{CrC}_{n}^{+}$, recouper les résultats obtenus pour les ions positifs.

Signalons qu'une étude par source à étincelles a été faite pour les $\mathrm{ZrC}_{n}^{+}$qui montre une prédominance des espèces à nombre pair d'atomes [19]. Ce résultat peut s'expliquer dans le cadre précédent. Le zirconium a une structure électronique externe $4 d^{2} 5 s^{2}$; il est donc normal de considérer que dans la molécule $\mathrm{ZrC}_{n}$, il conserve 4 électrons atomiques sur une couche $\mathrm{d}$; dans ces conditions, il ne fournit pas d'électrons à la couche $\pi$ dont le remplissage se fait de la même façon que dans les agrégats $\mathrm{CrC}_{n}$ (Fig. 3b). Comme pour ces derniers, les énergies d'ionisation sont plus faibles pour $n$ pair, ce qui est bien en accord avec le résultat expérimental.

\section{Bibliographie}

[1] Joyes, P., Leleyter, M., C.R. Hebd. Séan. Acad. Sci. B 274 (1972) 751-4.

[2] Bourguillot, R., Cornu, A., Massot, R., Pellet, J., Rapport CENG/DPC/IS/SSM 63-235 JG (1964) Grenoble.

[3] Joyes, P., J. Phys. Chem. Solids 32 (1971) 1269-75.

[4] Leleyter, M., Joyes, P., Radiat. Eff. 18 (1973) 105-110.

[5] Leleyter, M., Joyes, P., J. Phys. B 7 (1974) 516-528.
[6] Leleyter, M., Thèse d'Etat (1975) Orsay.

[7] Leleyter, M., Joyes, P., J. Physique 36 (1975) 343-55.

[8] Leleyter, M., Joyes, P., J. Physique Colloq. 38 (1977) C2-11 à $\mathrm{C} 2-17$.

[9] Können, G. P., TIP, A., DE VRIES, A. E., Radiat. Eff. 21 (1974) 269-74;

Können, G. P., Thèse (1974) Amsterdam, Pays-Bas. 
[10] Garrison, B. J., Winograd, N., Harrison, D. E. Jr., J. Chem. Phys. 69 (1978) 1440-4.

[11] Vidal, G., Galmard, P., Lanusse, P., Mém. Sci. Rev. Métall., tome LXV no 11 (1968).

[12] Pitzer, K. S., Clementi, E., J. Am. Chem. Soc. 81 (1958) 4477 85.

[13] Palmer, H. B., Shelef, M., Chemistry and Physics of Carbon (Marcel Dekker Inc., New York), édité par Walker, P. L. Jr., 4 (1968), pp. 85-135.

[14] Honig, R. E., Advances in Mass Spectrometry (Pergamon Press, Oxford) 2 (1962) 25-37.
[15] Mulliken, R. S., Person, W. B., Molecular Complexes (Wiley Interscience) 1969, p. 281.

[16] National Bureau of Standards, Ionization Potentials, NSRDSNBS 26.

[17] Anderson, A. B., J. Chem. Phys. 64 (1976) 4046-55.

[18] ORgel, L. E., An Introduction to Transition Metal Chemistry. Ligand field Theory (Methuen \& co., Londres) 1963, Chap. 3.

[19] CooK, H. D., Proc. 12th Ann. Conf. Mass Spectrom. and Allied Topics (1966) 133-7.

[20] SALEM, L., The Molecular Orbital Theory of Conjugated Systems (Benjamin, New York) 1966. 